\title{
Planning Travel as Everyday Design
}

\author{
Dean M.G. Hargreaves \\ University of Technology, Sydney. \\ Broadway, Ultimo, Sydney, NSW 2007 \\ dean@it.uts.edu.au
}

\author{
Toni Robertson \\ University of Technology, Sydney \\ Broadway, Ultimo, Sydney, NSW 2007 \\ toni@it.uts.edu.au
}

\begin{abstract}
This paper examines the implications of conceptualising planning as a type of design activity. This is explored through results from a two-month field study that investigated the planning and decision making behaviour of people engaged in preparing for multipoint, international air travel. Planning travel is a type of ill-structured complex problem that is characterised as being temporally sporadic, sometimes synchronous, often asynchronous, frequently collaborative, and spatially varied with participants at different times co-located and in separate places. Research participants were professional travel agents and non-professional but experienced travel planners. Ancillary material collected included photographs of the planning situation and drawings and notes made by participants. In contrast to the formalised prescriptive planning models common in cognitive science and operations research, the everyday planning activity featured in this study is situated and naturalistic. This research is undertaken with a view to designing systems to support the design and decision making activity of travel planners.
\end{abstract}

\section{Author Keywords}

Planning, decision making, planning support.

\section{ACM Classification Keywords}

H5.m. Information interfaces and presentation (e.g., HCI): Miscellaneous.

\section{INTRODUCTION}

This paper introduces and discusses the potential for applying design theory to planning. This is explored through travel planning activity that is framed as a type of design process and is situated within the context of everyday life. The Oxford English Dictionary defines a plan as 'an organised (and usually detailed) proposal according to which something is to be done'. The New Oxford American Dictionary defines a plan as 'a detailed proposal for doing or achieving something'. Both these definitions locate the plan prior to the action. In these cases action takes the form of the implementation of the plan. Both definitions describe the efficacy of the plan in terms of its level of detail in describing the future action, therefore, the more detailed the plan prior to action the better the plan. This view represents the classical planning models used within cognitive science and operations research (OR). Classical plans are typically represented as sequences of actions designed to accomplish well defined goals (Suchman, 1987). This represents an idealised planning model and is prevalent in 'first-generation sys-

OZCHI 2010, November 22-26, 2010, Brisbane, Australia. Copyright the author(s) and CHISIG

Additional copies are available at the ACM Digital Library

(http://portal.acm.org/dl.cfm) or ordered from the CHISIG secretary (secretary@chisig.org)

OZCHI 2010 Proceedings ISBN: 978-1-4503-0502-0 tems' approaches within OR (Rittel and Webber, 1973). This typically includes variations of the following sequence: identifying problems, developing systematic procedures, exhaustively evaluating alternative strategies and action sets, and statistically monitoring conditions and feedback to help formulate new goals and actions (Rittel, 1972). Where the objective is to define optimised paths toward clearly defined goals.

This model has demonstrated effectiveness for certain types of structured problems, usually encountered in the natural sciences and engineering (Rittel and Webber, 1973), which can be reduced to systematic computational routines (Simon and Newell, 1958). However, this model was found to be vastly inadequate for dealing with classes of problems that cannot easily be defined or structured. Simon and Newell (ibid) elaborated a distinction between what they termed well-structured problems and ill-structured problems. Well structured problems were amenable to the classical view of planning, and in such situations were deployed very successfully. Simon and Newell (ibid) identified that while the systematic application of scientific techniques to well structured problems had resulted in great advances, they were much more difficult to apply to the ill-structured problems that required human judgement and heuristic thinking processes. In short, the vast majority of problems that people encountered as part of everyday life. Their suggested solution was to develop computational systems that emulated this heuristic thinking and judgment (Simon and Newell, 1958). This solution rested on the quantification of these heuristics to enable them to be interpreted by computers. As is now well documented, this was a much more challenging undertaking than Simon and Newell (ibid) had envisioned and as a result very little progress has been made on this project since (Dreyfus, 1986).

Rittel (1972) provides an alternative to the computational route through a substantial reconceptualisation of illstructured problems as 'wicked' problems. Like Simon and Newell (1958), Rittel (1972) claims that planning within human systems, for example policy formulation or large-scale community based engineering works, are a different category of planning problem to those confronted in the natural sciences. Wicked problems are often difficult to define as they frequently consist of multiple competing goals, some of which are declared and others undeclared; their goals may be redefined or may emerge as the activity progresses or as new information becomes available, and; they may be multiple competing solutions. Wicked problems have the following properties: each new problem is essentially unique; each problem becomes a symptom of another problem; improving aspects of the world in which people live replaces a search for truth as an intent. 
Rittel (1973) proposes a 'second generation' OR within which he suggests an approach based on an argumentative process, through which a version of the problem and potential solutions emerge among participants. This is driven by the structured exercise of human judgment and critical argument among participants. This approach shares many similarities to Checklands' (1981) systems thinking and his formalisation of this as soft-systems methodology. Checkland (ibid) suggested a shift in planning and problem solving from approaches based in classic first generation OR with its emphasis on computationally based optimisation, to an approach featuring a focus on human experience, judgment and learning. The goal became improving the performance of systems through sequential interventions guided by structured cycles of reflective action in real-world settings that promoted ongoing learning about the system (Checkland, 1981).

Travel planning is a type of joint planning activity where multiple nascent intentions are formed, communicated, and modified concurrently with information gathering, communication with experts and peers and the use of planning tools. These plans are revised and revisited in ways that are highly responsive to the situation and become generative of themselves. For most travel planning the goal is to develop an itinerary with some level of specification. Travel planning is particularly interesting as a planning exercise, as the output is often a detailed plan, which is later enacted as travel. It could be argued that this conforms to a classical planning mode where detailed planning precedes action. While this may be true, it is argued here that the planning process itself that generates the itinerary is an emergent planning activity that can helpfully be thought of as a design process.

The next section reports on a field study that investigated the travel planning activity of professional and nonprofessional but experienced travel planners.

\section{FIELD STUDY}

To better understand people's planning and decision making activity, with respect to travel, a field study was conducted over a two month period. Field visits were undertaken to travel agents, and to the the homes of nonprofessional travel planners, to collect ancillary data. This included taking photographs of the primary planning environment, noting planning tools such as whiteboards, manuals, computers or notepads. People selected for interviews were those who had recently returned from a trip, or who were in the process of planning travel that involved a degree of complexity. This usually included a multi-stop international trip with a combination of different airlines or ground sectors. Interviewees were selected using a convenience sampling approach (Ferber, 1977). As such the sample was drawn from those people who were close to hand. Referrals to other interviewees were then requested from the initial research participants. Whilst the results from a convenience sampling approach are not typically considered generalisable, the results nonetheless provided helpful insights that guided data collection, helped develop the research questions, and contributed to developing low-fidelity prototypes.

The interviews were semi-structured, and were audio recorded with text transcripts produced for further analysis. Interviews were typically an hour to an hour and half in length. Materials, tools and documents that were used as part of the planning process were scanned or photographed. The data included photographs of the layout of each travel agents desk, notes or other information attached to computer monitors or nearby walls, and the spatial relationship of the various tools. Other items included handwritten notes from a travel agent that were created during a phone conversation with a client, annotations to these, whiteboard planning notes and diagrams, and itineraries provided to travellers by travel agents.

In order to identify a provisional set of themes, an initial open-ended pilot interview was conducted with two people who were known to be frequent travellers. Each participant was asked about their general experiences in planning travel, focusing on felt frustrations or difficulties. A number of issues emerged from these interviews that would form the basis of an interview instrument. These interviews evolved over time as more was learnt about issues raised by research participants. Subsequently, 16 face-to-face interviews were completed in two separate rounds of interviews. These were conducted with people currently planning travel, professional travel agents, and nonprofessional travel planners. The first round of interviews was conducted with ten subjects using the interview instrument. A second round of interviews was then undertaken with a further six subjects to validate these findings.

The handwritten notes and diagrams collected during the field study were central to understanding how people planned their travel. These notes were created by planners during the early stages of planning a travel event and were remarkably similar between professional and nonprofessional planners. By visually grouping data contained in these hand drawn notes, common types of information, activities and processes started to emerge. This data was then compared to and validated against the data collected through subsequent interviews, and analysed based on the principles of grounded theory. As such it featured elements of open coding (Strauss, 1987) as a way to identify themes that were likely to be significant to the planning process. In an open coding mode, data is analysed in a granular fashion - usually line-by-line with the aim of generating concepts that fit the data. These concepts or categories are provisional and changed as new data became available. The themes identified during these interviews are outlined below.

\section{THE PROCESS OF PLANNING TRAVEL}

A number of concepts or categories are drawn from the data, which describe the processes people undertake when planning complex travel. These include: the emergence and identification of an initial motivating event; seeking inspiration and conducting preliminary research about destinations; drafting a destination sequence; allocating dates, durations and activities to each destination; creating a draft itinerary; researching accommodation and activities for each destination; undertake several iterations of the itinerary; completing budget and further iteration, and; sharing the itinerary with family, friends and colleagues. These are discussed further below.

\section{Emergence of an initial motivating event}

A motivating event is the initial reason people decide to embark on travel. Some motivating events identified included: concerts, conferences, workshops, holidays or 
sporting trips. Typically a motivating event is the centrepiece of the trip around which other activities are planned. While a motivating event can be a reason for travel it also places constraints on other aspects of the trip. For instance, motivating events were often work related (eg. a conference) - and the planning activity necessitated accommodating leisure activities around these work commitments and constraints.

\section{Seeking inspiration and preliminary research}

This phase is typified by broad and ill-structured search for information that may inspire ideas as part of the later planning. Sources of information included books (both fiction and non-fiction), magazines and newspaper articles, stories from friends, Internet searches, maps, atlases and blogs. Material that inspired people's thinking included physical sources and online resources. Another resource was existing itineraries published by travel agents. Examples were provided where inspiration was drawn from fiction novels, short stories, films, and other artistic references. People relied heavily on other travellers experiences, particularly people who the travel planner knew personally or regarded highly.

\section{Drafting a destination sequence}

Travel planners produce a draft destination sequence very early in the planning process. This involves a sequential list, quite often abbreviated, of the destinations that the traveller desires to visit. Often this list does not include dates or days of the week, rather just a list of places or things that the traveller would like to see or do. This list tends to be tentative and often aspirational.

\section{Allocating dates, durations and activities}

After destinations have been identified and placed in sequence, travel planners then assign dates, activities and durations to each destination. This is usually expressed sequentially and often involved an abbreviated representation of the day of the week e.g. M for Monday, T for Tuesday etc. The durations between stops were sometimes recorded here; for instance, driving time from Johannesburg to Venda in South Africa; or flight time from Johannesburg to London. Priorities for activities and destinations and length were also changed here. Research on activities begins here, which informs priorities e.g. if there are three sites of interest at a destination then the stay may be extended.

\section{Create a draft itinerary - building trip scenarios}

Planners then began to develop an itinerary and created scenarios to evaluate trade-offs. These different scenarios were retained and often referred to later in the planning process. Online travel websites were often used to learn about flight routes and costs before contacting a physical travel agent. Interviewees indicated they didn't 'trust' travel agents to find them either the best price or route. Consultants at low-cost travel agents, work with narrow margins in order to minimise time taken on each booking, as they are rewarded commission per sale made.

\section{Research for accommodation and activities}

This is a detailed search for accommodation and activities at each destination. This included recording price information against activity, and detailed comparison of accommodation options. The sources of information included: travel websites, travel agents, friends, web searches, blogs, fictional and biographical accounts and other sources that were viewed as credible and authoritative. This was viewed as a time consuming and frustrating activity. User accommodation reviews on sites such as TripAdvisor.com were viewed as biased toward 'complainers' i.e. people with grievances are motivated to contribute more than those who were happy.

\section{Iterate itinerary - additional scenarios}

The itinerary is then revised based on information gathered during the accommodation and activity search. For example, planners change the itinerary if accommodation is problematic, (eg a major event occurring), or if there are more interesting alternatives nearby. This often leads to revising scenarios.

\section{Draft budget and additional iteration}

Planners then construct a draft budget. The itinerary is iterated by evaluating trade-offs between activities, accommodation, duration and flights. This featured close collaboration with other co-travellers. Up to this point there are often between 5 and 15 iterations of the itinerary. An iteration of the itinerary at this stage refers to how many times the travel agent or traveller produces a new version in response to changed requirements, or to accommodate a new constraint.

\section{Share itinerary}

This includes developing a version of the itinerary that travellers provide to friends and family, people that they are likely to stay with, or to provide to their organisation. People rarely supplied just the basic itinerary provided by the airline, website or travel agent; the itinerary was often annotated with hotel or accommodation information, car hire, notes about activities, maps, contact information at each destination, including phone numbers and addresses, and even the names of people that they might be staying with. The reasons provided for distributing this version of the itinerary included safety and security, courtesy and practical reasons that included meeting people at certain places. This additionally provided a formal record for an organisation for business travel - in order to comply with health and safety policies, and for reporting and accountability purposes.

\section{Observations on the travel planning phases}

While it proved immensely helpful to categorise the data in this way, it should be acknowledged that the boundaries between each category varied between planners, with iteration often occurring within and between several of the categories. Despite the fluidity of categories, this structure has proved useful in explaining peoples planning activity.

\section{DISCUSSION: PLANNING AS DESIGN}

This field study illustrates the extent to which travel planning is a social and collaborative activity, in contrast to the idea of an individual working largely alone, and engaged primarily in an optimisation process. This activity more often than not involves several people at different locations. As might be expected this necessitated frequent communication, which was undertaken not surprisingly by email, telephone and face-to-face. Email was the most commonly used tool, with in one instance more then 100 emails sent between two participants involved in a joint planning exercise.

What if we are to frame the travel planning process as a design activity? To help understand the potential for this, 
we first look to Alexander (1982) who calls for applying the methods and processes of design to decision-making within organisations, specifically for the formulation of public-policy. Alexander (ibid) draws on Simon's description of design as 'a process of devising courses of action to move from existing situations to preferred ones.' Alexander (ibid) describes two categories of decision-making: the first conceptualises decisionmaking as a process of searching for and choosing between alternative solutions that already exist. This approach has demonstrated effectiveness for certain wellstructured problems where alternative potential solutions can be described in advance and evaluated in terms of achieving stated goals. This however becomes problematic for wicked problems where neither the problem or potential solutions can be easily described. Alexander (ibid) describes a second type of decision making where the focus shifts from searching for, evaluating and then implementing pre-existing solutions to generating solutions where none previously exist. This he argues is a type of design activity. These two types of planning can be applied to different types of travel planning.

The type of 'packaged' travel where non-professional planners are only required to choose between a limited set of destinations, accommodation and flight options might well be described by Alexanders (ibid) search category. A second type of travel planning is significantly more complex and involves planners generating solutions where none of the existing solutions are adequate. The type of travel planning described in the field study involves both search (e.g. choosing between available flights for a given sector) and design approaches (e.g. what route should I take?). Creating itinerary scenarios involves making a series of tentative decisions that then allow people to explore consequences (usually against cost and time constraints) of different courses of action. This paper argues that the process of developing an itinerary is essentially a planning activity, with the itinerary itself the primary outcome. The planning process requires planners to make decisions among alternatives at many different stages. The itinerary is produced generatively as a result of these decisions. Here the decision making process is located within the planning activity, and not as an endpoint of that process. It is for this reason that this paper provides a focus on the centrality of planning, and specifically of planning as a design activity.

Planning travel in the context of everyday activity does not conform to a classical linear planning model. The classical model is described with various degrees of critical appraisal by Suchman (1987), Alexander (1982) and Simon (1958) as a systematic sequential activity, with a focus on well-structured problems to what is described here as design activity with a focus on learning and generating solutions to wicked problems. This paper argues that planning travel is helpfully re-conceptualised as a design activity that is iterative, collaborative, social and at times partial and embedded deeply in the context of everyday life.

\section{Future work: systems that support designing plans}

Such is the proliferation of packaged travel, that computer systems have been developed to support people to search and choose amongst the multitude of various packaged options. These systems fall under a category known generally as recommender systems. These systems allow users to enter personal preferences, to specify broad travel types, select from categories of destinations (eg beach, country, adventure etc), and draw on a person's previous travel history (Resnick and Varian, 1997). The software then provides recommendations for travel packages that meet user entered criteria. These systems were developed for online travel agencies and many have since been discontinued. Readers would be familiar with Amazon's online book recommendations, which is one of the better known recommender systems. These systems support search, as described by Alexander (1982) above, and decision making amongst generic alternatives. However, by virtue of the kinds of decisions they are designed to assist, they provide very little support for design aspects of the planning process.

In contrast, this research explores the potential to develop systems that support the social, communicative, collaborative and design aspects of the planning process. The next phase of this research will involve iteratively developing and evaluating a prototype together with research participants to support designing plans.

\section{CONCLUSIONS}

New theories of planning reflect a shift from classical linear planning models to iterative, deeply contextual models. This paper proposes applying recent advances in design theory to planning in the context of everyday life. These issues were explored through a two-month field study of people engaged in multi-stop international travel, which we understand to be a complex, social and collaborative process. This field study reveals that planning travel occurs both synchronously and asynchronously, is temporally sporadic and involves multiple parties in the interaction. Physical and digital tools are used to support planning, communication and co-ordination. Future research will explore the development of systems to support this activity.

\section{REFERENCES}

Alexander, E. 1982. Design in the decision-making process. Policy Sciences, 14, 279-292.

Checkland, P. 1981. Systems thinking, systems practice, Wiley.

Dreyfus, H. L. 1986. Mind over Machine: The Power of Human Intuition and Expertise in the Era of the Computer. Cabinet 1-230.

Ferber, R. 1977. Research by convenience. Journal of Consumer Research, 57-58.

Resnick, P. \& Varian, H. 1997. Recommender systems. Communications of the ACM, 40, 58.

Rittel, H. 1972. On the planning crisis: systems analysis of the first and second generations. Bedrifts Okonomen, 8, 390-396.

Rittel, H. \& Webber, M. 1973. Dilemmas in a general theory of planning. Policy Sciences, 4, 155-169.

Simon, H. \& Newell, A. 1958. Heuristic problem solving: The next advance in operations research. Operations Research, 6, 1-10.

Strauss, A. 1987. Qualitative analysis for social scientists, Cambridge Univ Pr.

Suchman, L. 1987. Plans and situated actions: The problem of human-machine communication, Cambridge University Press. 